NBI-HE-96-04

January 31, 1996

\title{
Correlation Functions in the Multiple Ising Model Coupled to Gravity
}

\author{
M. G. HARRIS and J. AMBJØRN \\ Niels Bohr Institute, Blegdamsvej 17, \\ DK-2100 Copenhagen, Denmark. \\ E-mail addresses: Martin.Harris@nbi.dk, ambjorn@nbi.dk
}

\begin{abstract}
The model of $p$ Ising spins coupled to $2 \mathrm{~d}$ gravity, in the form of a sum over planar $\phi^{3}$ graphs, is studied and in particular the two-point and spin-spin correlation functions are considered. We first solve a toy model in which only a partial summation over spin configurations is performed and, using a modified geodesic distance, various correlation functions are determined. The two-point function has a diverging length scale associated with it. The critical exponents are calculated and it is shown that all the standard scaling relations apply. Next the full model is studied, in which all spin configurations are included. Many of the considerations for the toy model apply for the full model, which also has a diverging geometric correlation length associated with the transition to a branched polymer phase. Using a transfer function we show that the two-point and spin-spin correlation functions decay exponentially with distance. Finally, by assuming various scaling relations, we make a prediction for the critical exponents at the transition between the magnetized and branched polymer phases in the full model.
\end{abstract}

\section{Introduction}

The work of Knizhnik, Polyakov and Zamolodchikov (KPZ) [1] as well as of David, Distler and Kawai (DDK) [2] made it possible to understand many aspects two-dimensional quantum gravity coupled to conformal field theories. At the same time, it became clear that the models of dynamical triangulations coupled to matter fields provide us with a statistical mechanical realisation of the models described by KPZ and DDK, in the same way as the two-dimensional Ising model at its critical point can be viewed as a conformal 
$c=1 / 2$ field theory. Two aspects of $2 \mathrm{~d}$ quantum gravity coupled to matter fields remain puzzling: the $c=1$ barrier and the concept of correlation length. The formulae for critical exponents derived by KPZ cease to be valid for $c>1$ and the critical exponents are derived by general scaling arguments applied to globally defined operators. At no point is the concept of a divergent correlation length introduced. For ordinary statistical systems the divergence of a correlation length when a critical temperature is approached is believed to be the underlying reason that general scaling arguments work well. The difficulties of defining a local length scale in quantum gravity are well known and have so far prevented a proper treatment of correlation functions by means of continuum methods.

Working entirely in the context of dynamical triangulations the problems mentioned are not seen directly. Statistical models with multiple Ising spins living on dynamically triangulated surfaces are perfectly well defined even if $c>1$ and they have a critical point. At least superficially, the only difference compared to $c<1$ is that we cannot solve the theory. Nevertheless, low temperature expansions and mean field calculations seem reliable for $c \rightarrow \infty$, as is confirmed by the agreement between the theoretical calculations and Monte Carlo simulations of the systems with large $c$ [3, 5]. The picture which emerges for large $c$ from the low temperature expansion [- $\mid$ is as follows: for large $\beta$ (low temperature) there is a magnetized phase for which $\gamma_{s t r}=-1 / 2$ separated from a branched polymer phase, where $\gamma_{s t r}=1 / 2$, by a transition at a critical $\beta^{*}$ where $\gamma_{s t r}^{*}=1 / 3$.

Likewise it has been possible by the use of dynamical triangulations to address the question of correlation length in two-dimensional quantum gravity. A two-point correlator between "punctures" has been calculated in pure gravity as a function of a "quantum" geodesic distance and it is found that standard scaling relations, known from the theory of critical phenomena, are satisfied, although with unusual critical exponents [11]. So far it has not been possible in $2 \mathrm{~d}$ quantum gravity to calculate correlation functions of matter fields as a function of the geodesic distance. The attempts to measure the spin-spin correlation functions by Monte Carlo simulations and to define a divergent correlation length as one approaches the critical point, have so far been ambiguous [16, 3]. The question arises as to whether there is a divergent spin-spin correlation length associated with the phase transition between a magnetized and a non-magnetized phase in the twodimensional Ising model coupled to gravity. Rather surprisingly from a continuum point of view, we can, using dynamical triangulations, begin to answer this question in the limit of large $c$, i.e. in the limit where a large number of Ising models is coupled to quantum gravity.

The statistical model we will define and solve in the following sections is a toy model of $2 \mathrm{~d}$ quantum gravity coupled to Ising spins in the sense that the correct summation over all triangulations is performed, but not all the spin configurations are included. The spin configurations we include are precisely the ones which dominate in the large $c$ limit, 
at large $\beta$, namely those for which the domains are connected in a tree-like fashion with domain boundaries of minimal size. This model allows us to calculate the two-point functions (using a certain definition of distance, which is based on the geodesic distance) and extract the critical exponents. We will verify that these exponents satisfy standard scaling relations and that the geometrical interpretation of some of the exponents is related to the fractal structure of the underlying "space-time", in agreement with general arguments; that is, we explicitly verify that the exponent $\nu$ is related to the Hausdorff dimension by $\nu=1 / d_{H}$. The model has a third order transition between a tree-like (i.e. branched polymer) phase and a magnetized phase. It is found that there is a diverging correlation length in the tree phase associated with the geometric two-point function, but no diverging correlation length associated with the spin-spin correlation function.

Next we turn our attention to the full model of $p$ independent Ising spins coupled to $2 \mathrm{~d}$ gravity, which has a central charge of $c=p / 2$. Following the analysis of the toy model, we show that various two-point functions decay exponentially with distance in the magnetized phase. Again there is a diverging correlation length associated with the geometric two-point function. By assuming that all the standard scaling relations still hold for this model and making a few further fairly modest assumptions, we show that the critical exponents for the transition between the magnetized and branched polymer phases, in the full model, are the same as those in the toy model.

The rest of this article is organized as follows: in section 2 we define the toy model, define a variant of geodesic distance in the model and calculate the two-point function. In sections 3 and 4 the critical exponents and Hausdorff dimension are calculated for the toy model, whilst section 5 discusses the spin-spin correlation function. In section 6 we verify by direct calculation in an external magnetic field, that the magnetic exponents found by scaling arguments are indeed the correct ones. In section 7 we address some of the questions mentioned above for the full model, i.e. in the model where we sum over all the spin configurations. Finally, section 8 contains our conclusions.

\section{Toy model, without a magnetic field}

\subsection{Definition}

Before looking at the full model of $p$ spins coupled to $2 \mathrm{~d}$ gravity (in the form of a sum over planar $\phi^{3}$ graphs), we will solve a toy model, which is very similar to the one studied in [6]. As for that model we will show that there is a magnetized phase for which $\gamma_{s t r}=-\frac{1}{2}$ and a branched polymer phase with $\gamma_{s t r}=\frac{1}{2}$. On the boundary $\gamma_{s t r}^{*}=\frac{1}{3}$, which is the same value as that in [6, 7] and agrees with the result $\gamma_{s t r}^{*}=\bar{\gamma} /(\bar{\gamma}-1)$ for $\bar{\gamma}=-\frac{1}{2}$, the pure gravity value, which one might expect from the calculation in [8]. 
For our model we sum over all possible rooted planar $\phi^{3}$ graphs (let us denote this set of graphs by $\mathcal{G}_{r}^{I}$ ), but we only sum over a subset of the possible spin configurations, namely those for which the domains are connected in a tree-like fashion, with at most one link connecting any two domains. In this paper we shall only consider planar graphs, that is, $\chi=2$ throughout. Each vertex will be weighted with a factor of $x$ and has $p$ independent spins on it which can take the values \pm 1 (i.e. vertex $i$ has spins $S_{i}^{\alpha}$ on it, with $\alpha=1, \cdots, p)$. Links joining vertices with dissimilar spin configurations will be given a factor of $e^{-2 \beta}$ for each spin flavour which differs. Thus the grand canonical partition function, $\mathrm{T}$, is

$$
T=\sum_{G \in \mathcal{G}_{r}^{I}} x^{N} \sum_{\{S\}^{\prime}} \prod_{<i j>} \exp \left(\sum_{\alpha=1}^{p} \beta\left(\left\{S_{i}^{\alpha} S_{j}^{\alpha}-1\right)\right),\right.
$$

where the first summation is over graphs, with $N$ being the number of vertices in the graph. The product is over the nearest neighbour pairs on the graph $G$ (referred to as "links") and the second summation is over the following set of spin configurations. Take the graph $G$ and decompose it into a set of one-particle irreducible ("1PI") graphs (which we shall call "blobs") connected in a tree-like fashion (see fig. 1); note that these blobs are essentially just minimum necked baby universes ("minbu"s). The blobs are fully magnetized, that is, all the vertices within a 1PI blob have the same set of spins, and we will sum over the $2^{p}$ possible spin configurations for each blob (except the root blob for which all the spins will be fixed to be +1$)$.

Figure 1: Measuring distances

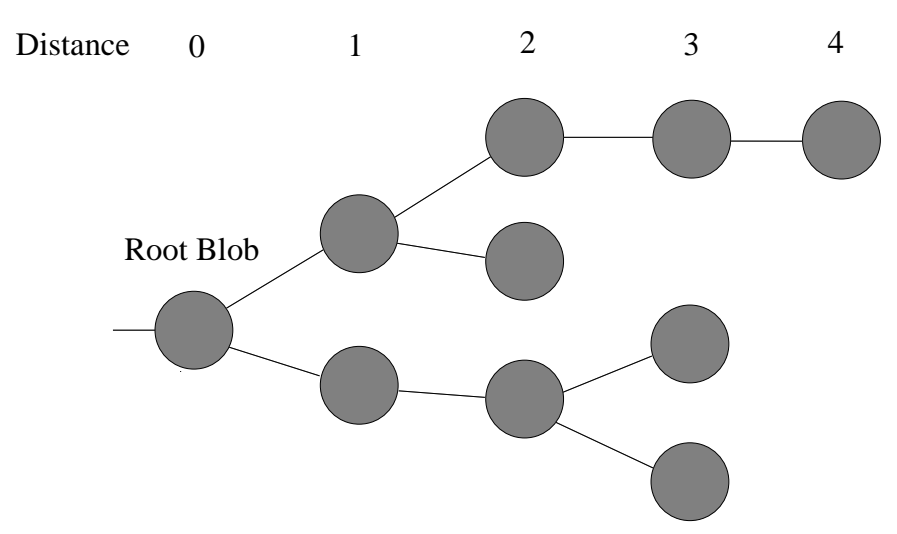

In the next sections we will solve this model exactly and also calculate various correlation functions, but in order to do this we need first to define how distances are to be measured on the graphs. One possible measure of the distance between two vertices $A$ and $B$, is the geodesic distance between them. That is, find the shortest path from $A$ to $B$ along links, counting a distance of one unit for every link traversed - this gives the geodesic distance between the two points. Unfortunately, this is quite difficult to deal 
with analytically, although some results are known for the pure gravity case [9]. In this paper we use a slightly different definition of distance, which is much simpler to handle. The distance between two vertices will be defined as the shortest path between them along links, counting a distance of one unit only for links that separate two 1PI blobs.

Since we are interested in correlations between the spins in the root blob, which are held fixed and spins further away, distances will be measured from the root blob. Thus all the vertices in the root blob have a distance zero, all the vertices within blobs connected by a single link to the root blob are at distance one and so on (see fig. 1). It will turn out that the average number of vertices in each blob is very small, so that for many graphs this definition of distance will be quite similar to the geodesic distance, especially in the branched polymer phase for which the partition function is dominated by tree-like graphs.

The advantage of measuring distances in this way is that it makes it very easy to define a transfer function, $f(y)$,

$$
f(y)=\sqrt{1-\lambda y} \mathcal{Z}^{I I}\left(x(1-\lambda y)^{-\frac{3}{2}}\right)+\frac{\lambda^{2}}{4 x} y^{2},
$$

where $\lambda=2 x\left(1+e^{-2 \beta}\right)^{p}$ and

$$
\mathcal{Z}^{I I}(x)=\sum_{G \in \mathcal{G}_{r}^{I I}} x^{N}
$$

This summation is over the set $\mathcal{G}_{r}^{I I}$ of rooted planar 1PI graphs.

This function, $f(y)$, takes a rooted 1PI blob and glues an arbitrary number of trees, each weighted with a factor of $y$, on to the blob. Note that each time we glue a tree on to a link in the blob, we pick up a factor of $x$, for the new vertex that is created, a factor of two because we can hang the tree in one of two directions and a factor of $\left(1+e^{2 \beta}\right)^{p}$ to take account of the $2^{p}$ different ways the spins on the blob and those on the tree's root blob can differ - this accounts for the factor of $\lambda$ multiplying each $y$. When we sum over all possible rooted 1PI blobs and all possible ways of gluing trees to links this gives the first term in (2). More specifically, the blob without any trees attached has a weight of $\mathcal{Z}^{I I}(x)$. Consider a rooted graph with $N$ vertices, it has $L=\frac{1}{2}(3 N-1)$ internal links and we can add an arbitrary number of trees to each link, giving a total contribution of $x^{N}\left(1+\lambda y+(\lambda y)^{2}+\cdots\right)^{L}$ for the graph. When we sum over all 1PI graphs this gives a contribution of

$$
\sum_{G \in \mathcal{G}_{r}^{I I}} x^{N}(1-\lambda y)^{-L}=\sqrt{1-\lambda y} \mathcal{Z}^{I I}\left(x(1-\lambda y)^{-\frac{3}{2}}\right) .
$$

The second term in (2) comes from the special case in which the root blob only consists of a single vertex, in which case we get a factor of $x$ for the vertex, $\left(1+e^{-2 \beta}\right)^{2 p}$ for the possible ways the spins may differ and $y^{2}$ for the two trees. 
Figure 2: $T=f(T)$

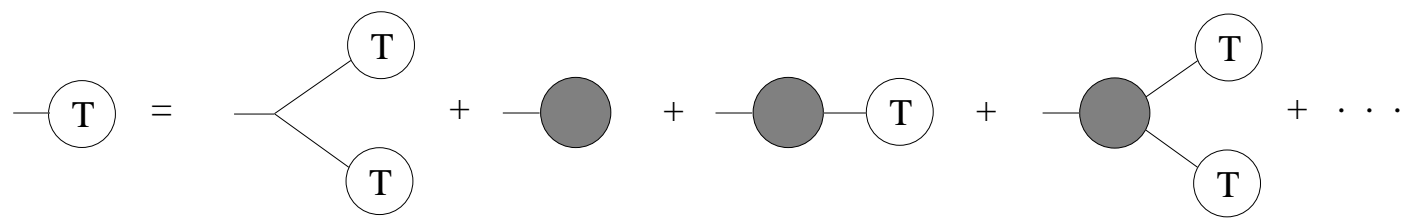

The partition function, $T$, satisfies $T=f(T)$ and this is represented diagrammatically in fig. 2; the shaded circle represents the sum over 1PI graphs, $\mathcal{Z}^{I I}(x)$. For fixed $p$ and $\beta$, this equation gives $T$ as a function of $x$. By finding the closest singularity to the origin, which occurs at $x_{c}(\beta, p)$, say, we can determine the free energy for the model. For what follows it will be convenient to define $x^{\prime}=x(1-\lambda T)^{-\frac{3}{2}}$. Now, there are essentially two different types of singularity. One occurs when $x^{\prime}$ equals $x_{c}^{I I}$, the critical value of $x^{\prime}$ for $\mathcal{Z}^{I I}\left(x^{\prime}\right)$ and this will correspond to the magnetized phase. The other occurs when the graphs become tree-like and we shall come back to this case later.

\section{$2.2 x_{c}$ in the magnetized phase}

Consider the first case, for which $x^{\prime}=x_{c}^{I I}$ and hence $\mathcal{Z}^{I I}\left(x^{\prime}\right)=\mathcal{Z}_{c}^{I I}$, then writing $T\left(x_{c}\right)$ as $T_{c}$,

$$
T_{c}=\sqrt{1-\lambda_{c} T_{c}} \mathcal{Z}_{c}^{I I}+\frac{\lambda_{c}^{2}}{4 x_{c}} T_{c}^{2}
$$

and $x_{c}^{I I}=x_{c}\left(1-\lambda_{c} T_{c}\right)^{-\frac{3}{2}}$, where $\lambda_{c}=2 x_{c}\left(1+e^{-2 \beta}\right)^{p}$. Defining,

$$
h \equiv\left(\frac{x_{c}}{x_{c}^{I I}}\right)^{\frac{1}{3}}=\sqrt{1-\lambda_{c} T_{c}}
$$

we get

$$
1-h^{2}=\left(1+e^{-2 \beta}\right)^{p}\left[2 x_{c}^{I I} \mathcal{Z}_{c}^{I I} h^{4}+\frac{1}{2}\left(1-h^{2}\right)^{2}\right] .
$$

However, $\mathcal{Z}^{I I}(x)$ is a known function, from Brézin et al [10] one can show that

$$
\mathcal{Z}^{I I}(x)=\frac{1}{x} \tau(1-3 \tau)
$$

where $x^{2}=\tau(1-2 \tau)^{2}$. This gives $\tau_{c}=\frac{1}{6}, x_{c}^{I I}=\sqrt{\frac{2}{27}}$ and $\mathcal{Z}_{c}^{I I}=\frac{1}{4} \sqrt{\frac{3}{2}}$. Hence we can solve (7) to get $x_{c}=x_{c}^{I I} h^{3}$ with

$$
h^{2}=\frac{3}{4 d}\left[d-1+\sqrt{\left(1-\frac{1}{3} d\right)(1+d)}\right]
$$

where we have defined $d \equiv\left(1+e^{-2 \beta}\right)^{p}$. This gives $x_{c}$ in the magnetized phase - we shall prove that it is magnetized later. 


\subsection{Number of blobs and $\gamma_{s t r}$}

Next we will calculate $\langle n\rangle_{r}$, the average number of 1PI blobs at distance $r$. Define $G_{r}=f^{(r)}(v T)$, where the notation means $f(f(f(\ldots f(v T))))$; this weights each blob at distance $r$ with an extra factor of $v$. Note that $v T$ can be regarded as the standard partition function, but with the root blob weighted by an extra factor of $v$. Applying the function $f$ to this gives $f(v T)$, where now each blob at distance one is weighted with the extra factor of $v$. Each application of $f$ just moves the weights of $v$ down the tree, by one unit of distance. Thus,

$$
\langle n\rangle_{r}=\left[\frac{v}{G_{r}} \frac{\partial G_{r}}{\partial v}\right]_{v=1}=\left.\frac{1}{T} \frac{\partial G_{r}}{\partial v}\right|_{v=1},
$$

where we have used that $G_{r}(v=1)=T$. However, $G_{r}=f\left(G_{r-1}\right)$ and thus

$$
\begin{aligned}
\langle n\rangle_{r} & =\frac{1}{T}\left[\left.\frac{\partial f}{\partial y}\right|_{y=G_{r-1}} \frac{\partial G_{r-1}}{\partial v}\right]_{v=1} \\
& =\left.\frac{\partial f}{\partial y}\right|_{y=T}\langle n\rangle_{r-1}=\left[\left.\frac{\partial f}{\partial y}\right|_{y=T}\right]^{r},
\end{aligned}
$$

since $\langle n\rangle_{0}=1$ (there is only one root blob). It will be convenient to define,

$$
B(x, \beta, p)=\left.\frac{\partial f}{\partial y}\right|_{y=T} .
$$

Using the formula (2) for $f(y)$, one can calculate $B$,

$$
B=\left.\frac{\partial f}{\partial y}\right|_{T}=\frac{\lambda}{2} \frac{\mathcal{Z}^{I I}\left(x^{\prime}\right)}{\sqrt{1-\lambda T}}\left(3 M^{I I}\left(x^{\prime}\right)-1\right)+2 x\left(1+e^{-2 \beta}\right)^{2 p} T
$$

where

$$
M^{I I}(x) \equiv \frac{x}{\mathcal{Z}^{I I}(x)} \frac{\partial \mathcal{Z}^{I I}}{\partial x}=\frac{1-\tau}{1-3 \tau} .
$$

Note that $M^{I I}\left(x_{c}^{I I}\right)=\frac{5}{3}$. Evaluating at $x_{c}$ will give $B_{c}(\beta, p)$. In the magnetized phase this gives us,

$$
B_{c}=1+\frac{1}{2}(d-1)-\frac{1}{2 \sqrt{3}} \sqrt{4-(d-1)^{2}} .
$$

Thus at $x_{c},\langle n\rangle_{r}=\left(B_{c}\right)^{r}$ and the average total number of blobs is

$$
\langle n\rangle=\frac{1}{1-B_{c}} .
$$

For large enough $\beta, B_{c}$ is less than one and positive, that is, the number of blobs decreases exponentially with distance. As $\beta$ is reduced, $B_{c}$ increases (since $\lambda$ increases and this encourages the tree to branch), until $B_{c}=1$ at some critical value, $\beta^{*}$; this corresponds to the boundary of the tree-like region. Using $T=f(T)$, one can show that,

$$
\frac{\partial T}{\partial x}=\frac{1}{1-B}\left[d^{2} T^{2}+\frac{\mathcal{Z}^{I I}\left(x^{\prime}\right)}{x \sqrt{1-\lambda T}}\left(M^{I I}\left(x^{\prime}\right)+x d T\left(M^{I I}\left(x^{\prime}\right)-1\right)\right)\right] .
$$


For $\beta>\beta^{*}, 0<B_{c}<1$ and $\left.\frac{\partial T}{\partial x}\right|_{x_{c}}$ is finite, whilst for $\beta \leq \beta^{*}, \frac{\partial T}{\partial x}$ diverges as $x \rightarrow x_{c}$. That is, $B_{c}=1$ throughout the entire tree-like region and $\gamma_{s t r}>0$ in this region, since $\frac{\partial T}{\partial x} \sim\left(x_{c}-x\right)^{-\gamma_{s t r}}$. One can easily calculate $\gamma_{s t r}$ in the various regions of the phase diagram. In the tree-like region and on the boundary, $\frac{\partial T}{\partial x} \sim \frac{1}{1-B}$, hence $1-B \sim(\Delta x)^{\gamma_{s t r}}$, where $\Delta x \equiv x_{c}-x$. However, we also have an expression (14) for $B$ and this gives $1-B \sim$ $(\Delta x)^{1-\gamma_{s t r}}$ inside the tree phase. Hence, $\gamma_{s t r}=\frac{1}{2}$ here as expected. On the boundary $1-B \sim(\Delta x)^{\frac{1}{2}\left(1-\gamma_{s t r}\right)}$ giving $\gamma_{s t r}^{*}=\frac{1}{3}$. In the magnetized phase $\frac{\partial^{2} T}{\partial x^{2}} \sim \frac{\partial^{2} \mathcal{Z}^{I I}\left(x^{\prime}\right)}{\partial x^{\prime 2}} \sim$ $\left(\Delta x^{\prime}\right)^{-\frac{1}{2}} \sim(\Delta x)^{-\frac{1}{2}}$, giving $\gamma_{s t r}=-\frac{1}{2}$ as expected.

Equation (16) applies in the magnetized phase and also on the boundary between the two phases. At $\beta^{*}, B_{c}=1$ and this gives using (16),

$$
\beta^{*}=-\frac{1}{2} \ln \left(2^{\frac{1}{p}}-1\right) .
$$

Note that this gives an estimate for the location, in the full model, of the transition between the tree and magnetized phases; compare this with the estimated location of the transition from the tree-like to the unmagnetized (pure gravity) phase, given in [12].

\section{$2.4 x_{c}$ in the tree phase}

Next we will calculate $x_{c}$ in the tree phase. At $x_{c}$ we have from $T_{c}=f\left(T_{c}\right)$,

$$
T_{c}=\sqrt{1-\lambda_{c} T_{c}} \mathcal{Z}^{I I}\left(x^{\prime}\right)+x_{c}\left(1+e^{-2 \beta}\right)^{2 p} T_{c}^{2}
$$

and from $B_{c}=1$, using (14),

$$
\frac{\lambda_{c}}{2} \frac{\mathcal{Z}^{I I}\left(x^{\prime}\right)}{\sqrt{1-\lambda_{c} T_{c}}}\left(3 M^{I I}\left(x^{\prime}\right)-1\right)+2 x_{c}\left(1+e^{-2 \beta}\right)^{2 p} T_{c}=1
$$

where $x^{\prime}=x_{c}\left(1-\lambda_{c} T_{c}\right)^{-\frac{3}{2}}$. Remembering that $d=\left(1+e^{-2 \beta}\right)^{p}$ and defining $X \equiv 1-\lambda_{c} T_{c}$, (21) gives

$$
\mathcal{Z}^{I I}\left(3 M^{I I}-1\right) x^{\prime} X d+2 d^{2} T_{c} x^{\prime} X^{\frac{3}{2}}=1
$$

and $(20)$ gives

$$
d^{2} T_{c}^{2} x^{\prime} X^{\frac{3}{2}}=T_{c}-X^{\frac{1}{2}} \mathcal{Z}^{I I}
$$

Thus,

$$
\mathcal{Z}^{I I}\left(3 M^{I I}-1\right) x^{\prime} X d+1-\frac{2}{T_{c}} X^{\frac{1}{2}} \mathcal{Z}^{I I}=0 .
$$

Moreover,

$$
T_{c}=\frac{1-X}{\lambda_{c}}=\frac{1-X}{2 x^{\prime} X^{\frac{3}{2}} d}
$$

and thus

$$
\mathcal{Z}^{I I}\left(3 M^{I I}-1\right) X(1-X) x^{\prime} d+1-X-4 x^{\prime} \mathcal{Z}^{I I} X^{2} d=0
$$


Also (23) gives

$$
4 x^{\prime} \mathcal{Z}^{I I} X^{2} d=(1-X)[2-d(1-X)] .
$$

Combining these last two equations gives

$$
\left[\mathcal{Z}^{I I}\left(3 M^{I I}-1\right) x^{\prime}-1\right] X d+d-1=0 .
$$

Using (8) and (15),

$$
X=\frac{d-1}{d(1-2 \tau)}
$$

and substituting into (27) gives

$$
\tau=\frac{1}{2}\left[1+2(d-1)^{2}\right]^{-1}
$$

and thus $x_{c}=x^{\prime} X^{\frac{3}{2}}$ is given by

$$
x_{c}=\frac{1}{2} d^{-\frac{3}{2}} \sqrt{d-1}
$$

in the tree phase. From equations (31) and (9) one can easily show that there is a third order phase transition with a finite discontinuity, that is, the critical exponent $\alpha=-1$.

\subsection{Number of vertices}

One can also calculate the average number of vertices at distance $r$. Define $G_{r}^{\prime}=$ $f^{(r)}\left(\left.f(T)\right|_{x z}\right)$, where the notation $\left.f(y)\right|_{x z}$ means the function $f(y)$, but with $x$ replaced everywhere by $x z$. Thus $\left.f(T)\right|_{x z}$ is just a tree, but with each vertex in the root blob weighted by an extra factor of $z$. Applying $f, r$ times, just pushes these weights down the tree to a distance $r$, so that in $G_{r}^{\prime}$ all the vertices at distance $r$ have an extra weight of $z$. Thus the average number of vertices at distance $r$ is given by

$$
\begin{aligned}
\langle N\rangle_{r} & =\left[\frac{z}{G_{r}^{\prime}} \frac{\partial G_{r}^{\prime}}{\partial z}\right]_{z=1}=\frac{1}{T}\left[\left.\frac{\partial f}{\partial y}\right|_{y=G_{r-1}^{\prime}} \frac{\partial G_{r-1}^{\prime}}{\partial z}\right]_{z=1} \\
& =B^{r}\langle N\rangle_{0}
\end{aligned}
$$

and this is essentially just the (geometric) two-point function (see section 4 ). The average total number of vertices is

$$
\langle N\rangle=\frac{\langle N\rangle_{0}}{1-B},
$$

where $\langle N\rangle_{0}$ is the average number of vertices in the root blob. This is given by

$$
\langle N\rangle_{0}=B+M^{I I}\left(x^{\prime}\right)-x d^{2} T\left(M^{I I}\left(x^{\prime}\right)+1\right) .
$$

In the magnetized phase this gives, at $x_{c}$,

$$
\langle N\rangle_{0}=\frac{4}{3}+\frac{1}{6}(d-1)+\frac{1}{2 \sqrt{3}} \sqrt{4-(d-1)^{2}}
$$


and in the tree phase,

$$
\langle N\rangle_{0}=\frac{2(d-1)}{(2 d-3)}
$$

Note that $d \equiv\left(1+e^{-2 \beta}\right)^{p}$, with $1<d<2$ in the magnetized phase and $d>2$ in the tree phase. The formulae also show that the average number of vertices in a blob is very small, for any values of $\beta$ and $p$; in fact $\langle N\rangle_{0}=2$ at $\beta^{*}$.

\section{Critical exponents from the scaling relations}

From equation (12) we see that there is an exponential decay of the number of blobs with distance. One can define a mass, $m$, in the model through,

$$
\langle n\rangle_{r}=B^{r}=e^{-m r}
$$

The mass is a function of $\beta, p$ and $x$. At $x_{c}$, as the critical line is approached from the magnetized phase, the mass vanishes (i.e. there is a correlation length equal to $1 / \mathrm{m}$, which diverges). Note that at $x_{c}, m=0$ throughout the whole tree phase. Let us use this definition of the mass and various scaling relations to calculate the critical exponents. Later we will calculate the magnetic exponents directly from the model, for the case $p=1$, gaining the same results for $\beta_{m}$ and $\delta_{m}$; this will show that our definition of the mass is reasonable and that the scaling relations hold for this toy model.

First let us consider the geometric exponents; note that we have already calculated $\gamma_{s t r}$ in section 2.3. The exponent $\nu$ is defined by $m \sim(\Delta x)^{\nu}$ for $\Delta x \rightarrow 0$ (where $\Delta x \equiv x_{c}-x$ ), but $m=-\ln B$ so that $m \sim 1-B \sim(\Delta x)^{\gamma_{s t r}}$ and hence $\nu=\gamma_{s t r}$; in the tree phase

$\nu=\gamma_{s t r}=\frac{1}{2}$ and on the boundary $\nu^{*}=\gamma_{s t r}^{*}=\frac{1}{3}$. Since there are no power law corrections to (38) we have $\eta=1$; note that at small $\Delta x$, for $1 \ll r \ll 1 / m$, one might expect [11] that $\langle n\rangle_{r} \sim r^{1-\eta}$. These sets of exponents satisfy Fisher's scaling relation $\gamma_{s t r}=\nu(2-\eta)$.

In reference [11] it is shown that if the two-point function has associated with it a vanishing mass, then for a suitable definition of the Hausdorff dimension, $\nu d_{H}=1$. This gives that $d_{H}=2$ in the tree phase, as we might expect for branched polymers and $d_{H}^{*}=3$ on the critical line.

Consider now the magnetic exponents, which we will write with a subscript $m$ to avoid confusion. Evaluating at $x_{c}$ and letting $\beta \rightarrow \beta^{*}$ from the magnetized phase, we have, defining $\Delta \beta \equiv \beta-\beta^{*}, m \sim \Delta \beta$ from (16) and hence $\nu_{m}=1$. If we take $\eta_{m}=1$ (we shall see later that the spin-spin correlation function has no power law corrections either), then using $\gamma_{m}=\nu_{m}\left(2-\eta_{m}\right)$ gives $\gamma_{m}=1$. Applying the other scaling relations $2-\alpha=\nu_{m} d_{H}^{*}, \beta_{m} \delta_{m}=\beta_{m}+\gamma_{m}$ and $\alpha+2 \beta_{m}+\gamma_{m}=2$, yields $\alpha=-1, \beta_{m}=1$ and $\delta_{m}=2$. The various exponents are listed in table 1. 
Table 1: Critical exponents

\begin{tabular}{|c||c|c|c|c|}
\hline Phase & $\gamma_{s t r}$ & $\nu$ & $\eta$ & $d_{H}$ \\
\hline Magnetized & $-\frac{1}{2}$ & & $(1)$ & \\
\hline Critical & $\frac{1}{3}$ & $\frac{1}{3}$ & 1 & 3 \\
\hline Tree & $\frac{1}{2}$ & $\frac{1}{2}$ & 1 & 2 \\
\hline
\end{tabular}

\begin{tabular}{|c|c|c|c|c|c|}
\hline$\alpha$ & $\beta_{m}$ & $\gamma_{m}$ & $\delta_{m}$ & $\nu_{m}$ & $\eta_{m}$ \\
\hline-1 & 1 & 1 & 2 & 1 & 1 \\
\hline
\end{tabular}

Before proceeding to check the magnetic exponents explicitly, we shall define $d_{H}$ and calculate it directly from the model.

\section{Hausdorff dimension}

As before in 11] we define the Hausdorff dimension, $d_{H}$, in terms of the two-point function

$$
T_{2}(r)=\sum_{G \in \mathcal{G}_{2}^{I}(r)} x^{N} W_{G}
$$

where $\mathcal{G}_{2}^{I}(r)$ is the set of planar $\phi^{3}$ graphs, with two marked points that are separated by a distance $r$. One of the marked points will be taken to be the vertex, in the root blob, which is connected to the external leg. $W_{G}$ is just the usual weight for the spin configurations, which appears in (11). Then $d_{H}$ is defined, in the continuum limit, by

$$
N(r) \sim r^{d_{H}}, \quad r \rightarrow \infty, \quad m(\Delta x) r=\text { const. },
$$

where

$$
N(r) \equiv \frac{1}{T_{2}(r)} \sum_{G \in \mathcal{G}_{2}^{I}(r)} N x^{N} W_{G}=x \frac{\partial\left(\ln T_{2}(r)\right)}{\partial x} .
$$

That is, by tuning $x$ to $x_{c}$ we are taking a continuum limit; however this definition of $d_{H}$ only really makes sense in the tree phase and on the boundary, where the mass vanishes at $x_{c}$.

Now $T_{2}(r)$ is just

$$
T_{2}(r)=\sum_{G \in \mathcal{G}_{r}^{I}} x^{N} W_{G} N_{r}=T\langle N\rangle_{r}=T B^{r}\langle N\rangle_{0},
$$

where $N_{r}$ is the number of vertices at distance $r$ for graph $G$;

$$
N(r)=x\left[\frac{1}{T} \frac{\partial T}{\partial x}+\frac{r}{B} \frac{\partial B}{\partial x}+\frac{1}{\langle N\rangle_{0}} \frac{\partial\langle N\rangle_{0}}{\partial x}\right] .
$$

As $r \rightarrow \infty$,

$$
N(r) \sim r \frac{\partial M^{I I}}{\partial x^{\prime}} \frac{\partial T}{\partial x} \sim r\left(x_{c}^{I I}-x^{\prime}\right)^{-\frac{1}{2}}(\Delta x)^{-\gamma_{s t r}}
$$


Since we are taking the continuum limit with $m r$ fixed, $r \sim m^{-1} \sim(\Delta x)^{-\gamma_{s t r}}$,

$$
N(r) \sim r^{2}\left(x_{c}^{I I}-x^{\prime}\right)^{-\frac{1}{2}}
$$

In the tree phase $N(r) \sim r^{2}$ giving $d_{H}=2$ and on the critical line $N(r) \sim r^{2}\left(\Delta x^{\prime}\right)^{-\frac{1}{2}} \sim$ $r^{2}(\Delta x)^{-\frac{1}{3}} \sim r^{3}$, so that $d_{H}^{*}=3$, as expected. Unfortunately, due to the way distances and the Hausdorff dimension have been defined in this model, $d_{H}$ is not well-defined in the magnetized phase. In reference [11] it is shown, using the geodesic distance, that $d_{H}=4$ for pure gravity (also the geometric exponents are $\nu=1 / 4$ and $\eta=4$ ) and this may well be true in the whole of the magnetized phase. It is certainly the correct value at $p=0$, where the toy model reduces to a pure gravity model.

Thus our definition of distance appears to correctly capture the behaviour of the model in the tree phase and on the critical line, where $\gamma_{s t r}>0$, but not within the magnetized phase.

\section{Spin-spin correlation function}

In this section we will calculate $\langle\Sigma S\rangle_{r}$ the average total spin at distance $r$; the summation is over all vertices at distance $r$ and all spin flavours on those vertices. Since the spins in the root blob are fixed to be +1 , this is essentially a spin-spin correlation function, for spins separated by a distance $r$. To calculate this we will add a magnetic field for vertices at distance $r$. First we will solve the model with $p=1$ and then the general case. Let us define

$$
f_{+}(y)=\sqrt{1-\lambda y e^{H}} \mathcal{Z}^{I I}\left(x e^{H}\left(1-\lambda y e^{H}\right)^{-\frac{3}{2}}\right)+x\left(1+e^{-2 \beta}\right)^{2} y^{2} e^{H},
$$

with $\lambda=2 x\left(1+e^{-2 \beta}\right)$. This is just $f(y)$, but with $x$ replaced by $x e^{H}$. The function takes a number of trees with weights $y$ and glues them on to a blob, where each vertex of the blob has a spin of +1 on it and there is a magnetic field $+H$ applied on these spins. The function $f_{-}(y)$ is defined in the same way, but with $H$ replaced by $-H$ throughout.

Let $T_{r}^{+}$be a tree with positive spins in the root blob and a magnetic field of $+H$ applied at distance $r$ from the root. Similarly $T_{r}^{-}$has spins of -1 in the root blob. Then $T_{0}^{+}=f_{+}(T)$ and $T_{0}^{-}=f_{-}(T)$. Define,

$$
F(y)=\sqrt{1-2 x y} \mathcal{Z}^{I I}\left(x(1-2 x y)^{-\frac{3}{2}}\right)+x y^{2} .
$$

Then

$$
\begin{aligned}
& T_{r}^{+}=F\left(T_{r-1}^{+}+e^{-2 \beta} T_{r-1}^{-}\right) \\
& T_{r}^{-}=F\left(T_{r-1}^{-}+e^{-2 \beta} T_{r-1}^{+}\right),
\end{aligned}
$$


that is, to make a graph with spins of +1 on the root blob and a magnetic field at distance $r$, one takes a number of trees with magnetic fields at distance $r-1$ and glues them on to a blob with positive spins, picking up a factor of $e^{-2 \beta}$ if the spins on the root of the tree are negative. Now,

$$
\langle\Sigma S\rangle_{r}^{+}=\left[\frac{1}{T_{r}^{+}} \frac{\partial T_{r}^{+}}{\partial H}\right]_{H=0}=\left.\frac{1}{T} \frac{\partial T_{r}^{+}}{\partial H}\right|_{H=0} ;
$$

the superscript on $\langle\Sigma S\rangle_{r}^{+}$denotes the fact that the vertices in the root blob have a single spin each, which is fixed to be +1 .

$$
\begin{aligned}
& \frac{\partial T_{r}^{+}}{\partial H}=\left.\left[\frac{\partial T_{r-1}^{+}}{\partial H}+e^{-2 \beta} \frac{\partial T_{r-1}^{-}}{\partial H}\right] \frac{\partial F}{\partial y}\right|_{y=T_{r-1}^{+}+e^{-2 \beta} T_{r-1}^{-}} \\
& \langle\Sigma S\rangle_{r}^{+}=\left.\left[\langle\Sigma S\rangle_{r-1}^{+}+e^{-2 \beta}\langle\Sigma S\rangle_{r-1}^{-}\right] \frac{\partial F}{\partial y}\right|_{y=\left(1+e^{-2 \beta}\right) T}
\end{aligned}
$$

but $\langle\Sigma S\rangle_{r-1}^{-}=-\langle\Sigma S\rangle_{r-1}^{+}$and $F(y)=f(2 x y / \lambda)$ so that

$$
\left.\frac{\partial F}{\partial y}\right|_{y=\left(1+e^{-2 \beta}\right) T}=\frac{2 x B}{\lambda} .
$$

Thus defining $t \equiv \tanh \beta$,

$$
\langle\Sigma S\rangle_{r}^{+}=B t\langle\Sigma S\rangle_{r-1}^{+}=(B t)^{r}\langle\Sigma S\rangle_{0}^{+}=(B t)^{r}\langle N\rangle_{0}
$$

Let us consider the general case of $p$ spin flavours for which $\lambda=2 x\left(1+e^{-2 \beta}\right)^{p}$,

$$
\begin{gathered}
T_{r}^{++\cdots+}=F\left(T_{r-1}^{+\cdots+}+e^{-2 \beta}\left(T_{r-1}^{+\cdots+-}+T_{r-1}^{+\cdots+-+}+\cdots\right)+\right. \\
\left.e^{-4 \beta}\left(T_{r-1}^{+\cdots+--}+\cdots\right)+\cdots+e^{-2 \beta p} T_{r-1}^{-\cdots-}\right) \\
\langle\Sigma S\rangle_{r}^{++\cdots+}=\frac{2 x B}{\lambda}\left[\langle\Sigma S\rangle_{r-1}^{+\cdots+}+e^{-2 \beta}\left(\langle\Sigma S\rangle_{r-1}^{+\cdots+-}+\cdots\right)+\cdots+e^{-2 \beta p}\langle\Sigma S\rangle_{r-1}^{-\cdots-}\right] .
\end{gathered}
$$

However one can easily show that,

$$
\langle\Sigma S\rangle_{r}^{\cdots}=\frac{1}{p}(p-2 n)\langle\Sigma S\rangle_{r}^{++\cdots+},
$$

where there are $p-n$ plus signs and $n$ minus signs in the superscript on the left-hand side. Thus,

$$
\begin{aligned}
\langle\Sigma S\rangle_{r}^{++\cdots+} & =\frac{B}{\left(1+e^{-2 \beta}\right)^{p}}\langle\Sigma S\rangle_{r-1}^{+\cdots+} \sum_{n=0}^{p} e^{-2 \beta n} \frac{1}{p}(p-2 n)\left(\begin{array}{c}
p \\
n
\end{array}\right) \\
& =(B t)\langle\Sigma S\rangle_{r-1}^{++\cdots+}=(B t)^{r} p\langle N\rangle_{0} .
\end{aligned}
$$


Now defining the magnetization at distance $r$ by

$$
\mathcal{M}_{r} \equiv \frac{\langle\Sigma S\rangle_{r}^{+\cdots+}}{p\langle N\rangle_{r}}
$$

we have that $\mathcal{M}_{r}=t^{r}$. It should be noted that if one uses the exponential decay of $\langle\Sigma S\rangle_{r}^{+\cdots+}$ or $\mathcal{M}_{r}$ to define a spin-spin correlation length, then this quantity will not diverge at the phase transition; this behaviour should be contrasted with that of the geometric correlation length, which does diverge.

If we define the total magnetization $\mathcal{M}$, for the case in which all the spins in the root blob are positive, by

$$
\mathcal{M} \equiv \frac{\langle\Sigma S\rangle^{+\cdots+}}{p\langle N\rangle}
$$

where $\langle\Sigma S\rangle^{+\cdots+}$ is the average of the total spin, which is a sum over all vertices and all spin flavours, then we have, at $x_{c}$,

$$
\mathcal{M}=\frac{1-B_{c}}{1-B_{c} t}
$$

Note that $\mathcal{M}=1$ for $\beta=\infty$, and that $\mathcal{M}=0$ throughout the tree phase, showing that this phase is unmagnetized. The other phase has $0<B_{c}<1$ and hence $0<\mathcal{M} \leq 1$; this is the magnetized phase as claimed earlier. Also at $x_{c}$, near $\beta^{*}, 1-B_{c} \sim \Delta \beta$, where $\Delta \beta \equiv \beta-\beta^{*}$, so that $\mathcal{M} \sim \Delta \beta$ and thus $\beta_{m}=1$, as calculated from the scaling relations. In fact for finite $p$,

$$
\mathcal{M}=\frac{4 p}{3} \Delta \beta+O\left((\Delta \beta)^{2}\right)
$$

so that the coefficient in front of $\Delta \beta$ is non-zero in general (note that $p=0$ would give $\left.\beta^{*}=-\infty\right)$.

\section{Toy model, with a magnetic field}

In the previous section we defined the magnetization in the grand canonical ensemble by (61). A different definition is possible namely,

$$
\mathcal{M}_{c e}=\lim _{N \rightarrow \infty} \frac{1}{p N}\langle\Sigma S\rangle_{N}
$$

where now we are working in the canonical ensemble, that is, we are using the set, $\mathcal{G}_{r}^{I}(N)$ of rooted $N$-vertex graphs.

In this section we will add a magnetic field, $H$, to the toy model. However, we shall only consider the case $p=1$, as $p>1$ appears to be more difficult to solve. It will be shown that the magnetic exponents $\beta_{m}$ and $\delta_{m}$ are the same for both definitions of the magnetization and agree with the results from the scaling relations. 
Rather than use the transfer function $f(y)$, which glues trees on to a 1PI blob, we shall use a different transfer function $\bar{f}(y)$, that glues trees on to a domain; this is more convenient for the $p=1$ model with a magnetic field. For $H=0$, it will give a sum over the same set of graphs that we had previously, with the same weights, but we will no longer be able to keep track of distances within the graphs.

Consider first the case $H=0$, the function $\bar{f}(y)$ is given by

$$
\bar{f}(y)=\frac{1}{\sqrt{1-2 x K}} \mathcal{Z}^{I}\left(x(1-2 x K)^{-\frac{3}{2}}\right)+x K^{2},
$$

where

$$
K=\frac{1}{2 x}(1-\sqrt{1-\mu y})
$$

with $\mu=4 x e^{-2 \beta}$ and

$$
\mathcal{Z}^{I}(x)=\sum_{G \in \mathcal{G}_{r}^{I}} x^{N}
$$

The function defined in (65) takes a rooted $\phi^{3}$ graph, which will form the core of a domain, and glues on trees weighted with factors of $K$. As before one picks up a factor of $2 x$ for each tree glued on. In this case however we allow trees to be glued on to the root link, which changes the power of the factor in front of $\mathcal{Z}^{I}$ compared with that in (2). This is necessary to make sure that we correctly sum over all possible domain structures.

The factor $K$ is the solution of

$$
K=e^{-2 \beta} y+x K^{2}
$$

This generates tree graphs whose vertices are weighted with $x$ and which have a factor of $e^{-2 \beta} y$ at the end of each branch. The domain corresponds to the core $\phi^{3}$ graph plus all the vertices in $K$. The ends of the branches in $K$, which we have weighted with $e^{-2 \beta} y$, correspond to the domain boundaries; setting $y=T$ will give us a set of domains glued together in a tree-like fashion.

Thus we have

$$
\bar{f}(y)=\frac{1}{(1-\mu y)^{\frac{1}{4}}} \mathcal{Z}^{I}\left(x(1-\mu y)^{-\frac{3}{4}}\right)+\frac{1}{4 x}(1-\sqrt{1-\mu y})^{2}
$$

and the grand canonical partition function (1) is the solution of $T=\bar{f}(T)$. This is shown diagrammatically in fig. 3 .

Note that this is just a different way of formulating the same model that we solved previously. One can easily use $T=\bar{f}(T)$ to calculate $x_{c}$ in the two phases, gaining the same results as before (to solve the $p$-flavour case one just uses $\mu=4 x(d-1)$ ). The advantage of this new formulation is that as we use $\bar{f}$ to move down the tree from one domain to the next, the sign of the spins alternates (at least for $p=1$ ). This simplifies 
Figure 3: $T=\bar{f}(T)$

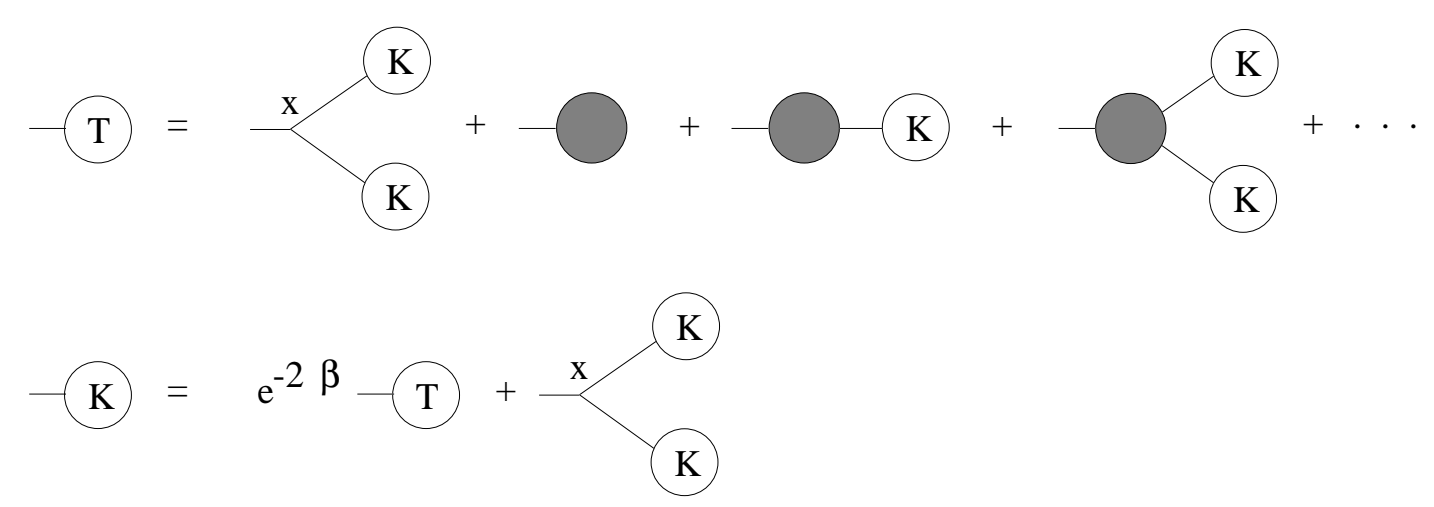

greatly the task of adding a magnetic field. For the original version of the model it is quite difficult to keep track of the spins on the different blobs.

Now let us add a magnetic field of $+H$, the transfer function which makes a domain with spins of +1 is

$$
\bar{f}_{+}(y)=\frac{1}{\left(1-\mu y e^{H}\right)^{\frac{1}{4}}} \mathcal{Z}^{I}\left(x e^{H}\left(1-\mu y e^{H}\right)^{-\frac{3}{4}}\right)+\frac{1}{4 x e^{H}}\left(1-\sqrt{1-\mu y e^{H}}\right)^{2} .
$$

Each spin in the domain is given an extra weight of $e^{H}$. It will be convenient to define $x_{+}^{\prime}=x e^{H}\left(1-\mu y e^{H}\right)^{-\frac{3}{4}}$. Similarly $\bar{f}_{-}(y)$, which makes a domain with negative spins is (70), but with $H$ replaced everywhere by $-H$. Let $T^{+}$be the partition function for graphs with positive spins in the root domain and $T^{-}$that for those with a negative root domain. Then $T^{+}=\bar{f}_{+}\left(T^{-}\right)$and $T^{-}=\bar{f}_{-}\left(T^{+}\right)$. These equations determine $T^{+}(x, \beta, H)$. For $H>0$ in the magnetized phase, the critical value of $x$ is determined by $x_{+}^{\prime}=x_{c}^{I}$, where $x_{c}^{I}$ is the critical value for $\mathcal{Z}^{I}\left(x_{+}^{\prime}\right)$. Defining

$$
\bar{h} \equiv\left(\frac{x_{c} e^{H}}{x_{c}^{I}}\right)^{\frac{1}{3}}
$$

we have

$$
T_{c}^{-}=\frac{1}{\mu_{c} e^{H}}\left(1-\bar{h}^{4}\right)
$$

and

$$
T_{c}^{+}=\bar{h}^{-1} \mathcal{Z}_{c}^{I}+\frac{1}{4 x_{c} e^{H}}\left(1-\bar{h}^{2}\right)^{2} .
$$

Note that $x_{c}^{I}=1 /\left(2.3^{\frac{3}{4}}\right), \mathcal{Z}_{c}^{I}=3^{\frac{3}{4}}\left(1-\frac{\sqrt{3}}{2}\right)$ and $M_{c}^{I}=1 /\left(\sqrt{3}-\frac{3}{2}\right)-1$ from [10]. However we still have $T^{-}=\bar{f}_{-}\left(T^{+}\right)$, and using this gives an equation determining $\bar{h}$. Putting

$$
X \equiv 1-\mu_{c} e^{-H} T_{c}^{+}=1-e^{-2 \beta} e^{-2 H}\left(1-\sqrt{3} \bar{h}^{2}+\bar{h}^{4}\right)
$$


and $x^{\prime}=x_{c} e^{-H} X^{-\frac{3}{4}}$, then we have

$$
e^{2 \beta}\left(1-\bar{h}^{4}\right)=e^{2 H}\left[1+X+2 \sqrt{X}\left(2 x^{\prime} \mathcal{Z}^{I}\left(x^{\prime}\right)-1\right)\right] .
$$

This gives $\bar{h}(\beta, H)$ and hence $x_{c}(\beta, H)$. Now,

$$
\mathcal{M}_{c e}(\beta, H)=-\frac{1}{x_{c}} \frac{\partial x_{c}}{\partial H}=1-\frac{3}{\bar{h}} \frac{\partial \bar{h}}{\partial H} .
$$

At $H \rightarrow 0$ we get by differentiating (75),

$$
\mathcal{M}_{c e}\left(\beta, H \rightarrow 0^{+}\right)=1-\frac{3\left(2-\frac{2}{\sqrt{3}} \bar{h}^{2}-\sqrt{3}(\bar{h})^{-2}\right)}{\left(3-\bar{h}^{2} \sqrt{3}-\bar{h}^{4}\left(2 e^{2 \beta}+e^{-2 \beta}+3\right)\right)},
$$

with

$$
\bar{h}^{2}=\frac{1}{2} \frac{1}{\left(1+e^{-2 \beta}\right)}\left[\sqrt{3} e^{-2 \beta}+\sqrt{4-e^{-4 \beta}}\right] ;
$$

note that at $H=0, \bar{h}^{2}=2 h^{2} / \sqrt{3}$, see equation (9). At $\beta$ close to $\beta^{*}=0$,

$$
\mathcal{M}_{c e}\left(\Delta \beta, H \rightarrow 0^{+}\right)=2 \Delta \beta+O\left((\Delta \beta)^{2}\right)
$$

Hence, $\beta_{m}=1$ as previously, but note that $\mathcal{M}_{c e}$ is not equal to $\mathcal{M}$. Now consider $\delta_{m}$, defined by $\mathcal{M}_{c e}\left(\beta^{*}, \Delta H\right) \sim(\Delta H)^{\frac{1}{\delta_{m}}}$. From $(\sqrt{75})$, one can show that $\mathcal{M}_{c e}$ is a certain function of $\bar{h}, e^{-2 \beta}, e^{2 H}, x^{\prime}, \mathcal{Z}^{I}\left(x^{\prime}\right), M^{I}\left(x^{\prime}\right)$ and $X$, where

$$
M^{I}\left(x^{\prime}\right) \equiv \frac{x^{\prime}}{\mathcal{Z}^{I}\left(x^{\prime}\right)} \frac{\partial \mathcal{Z}^{I}\left(x^{\prime}\right)}{\partial x^{\prime}} .
$$

At $\beta^{*}$ and small $\Delta H$, barring accidental cancellations, then all these functions are a constant plus terms of order $\Delta H$ (or smaller), except for $M^{I}\left(x^{\prime}\right)$,

$$
M^{I}\left(x^{\prime}\right)=M_{c}^{I}+\left(x_{c}^{I}-x^{\prime}\right)^{\frac{1}{2}}+\cdots .
$$

Since $x^{\prime}=x_{c}^{I}+O(\Delta H)$ then $M^{I}\left(x^{\prime}\right)=M_{c}^{I}+O\left((\Delta H)^{\frac{1}{2}}\right)$, giving $\delta_{m}=2$. Note that the value of $\delta_{m}$ depends crucially on the fact that $\gamma_{s t r}=-\frac{1}{2}$ in the magnetized phase.

Suppose that we next try to calculate $\gamma_{m}$, defined by

$$
\chi(\beta, H=0) \sim(\Delta \beta)^{-\gamma_{m}},
$$

then we find that $\chi \sim \frac{\partial^{2} x_{c}}{\partial H^{2}}$ and that this contains terms such as $\frac{\partial^{2} \mathcal{Z}^{I}\left(x^{\prime}\right)}{\partial x^{\prime 2}}$, which diverge as we take $H$ to zero. Thus $\gamma_{m}$ seems not to be well-defined, even though the scaling relations give $\gamma_{m}=1$.

Consider now the magnetization in the grand canonical ensemble, $\mathcal{M}$, defined by (61), $\beta_{m}$ has already been calculated and this just leaves $\delta_{m}$. We see that $\mathcal{M} \rightarrow 0$ at $\beta^{*}$, due to the divergence of $\langle N\rangle$,

$$
\left.\mathcal{M}\left(\beta^{*}, \Delta H\right) \sim \frac{1}{\langle N\rangle}\right|_{\beta^{*}}
$$


For graphs with a positive root domain, at $x_{c}$,

$$
\langle N\rangle^{+}=\left.\frac{x_{c}}{T_{c}^{+}} \frac{\partial T^{+}}{\partial x}\right|_{x_{c}}
$$

where $T^{+}=\bar{f}_{+}\left(\bar{f}_{-}\left(T^{+}\right)\right)$. This gives

$$
\frac{\partial T^{+}}{\partial x}\left[1-\left.\left.\frac{\partial \bar{f}_{+}}{\partial y}\right|_{T^{-}} \frac{\partial \bar{f}_{-}}{\partial y}\right|_{T^{+}}\right]=\left.\frac{\partial \bar{f}_{+}}{\partial x}\right|_{T^{-}}+\left.\left.\frac{\partial \bar{f}_{+}}{\partial y}\right|_{T^{-}} \frac{\partial \bar{f}_{-}}{\partial x}\right|_{T^{+}} .
$$

Thus

$$
\mathcal{M}\left(\beta^{*}, \Delta H\right) \sim 1-\left.\left.\frac{\partial \bar{f}_{+}}{\partial y}\right|_{T_{c}^{-}} \frac{\partial \bar{f}_{-}}{\partial y}\right|_{T_{c}^{+}}
$$

and again this is a function of various variables all of which are a constant plus $O(\Delta H)$, except that $M^{I}\left(x^{\prime}\right)=M_{c}^{I}+O\left((\Delta H)^{\frac{1}{2}}\right)$, giving $\delta_{m}=2$.

This completes our analysis of the toy model; we have derived all the critical exponents from the model (except for $\gamma_{m}$ ) and shown that all the usual scaling relations hold. Now we shall turn our attention to the full model of $p$-Ising spins coupled to $2 \mathrm{~d}$ gravity, and will find that many of the considerations in the previous sections apply for the transition between the tree and magnetized phases in this model.

\section{$7 \quad$ Full model}

\subsection{Definition}

Consider the full model where we have $p$ independent Ising spins on each vertex and are summing over all rooted planar $\phi^{3}$ graphs and all spin configurations. Then the partition function is, with $t=\tanh \beta$,

$$
\mathcal{Z}^{I}(x, \beta)=\sum_{G \in \mathcal{G}_{r}^{I}} x^{N}\left[\frac{1}{2^{N}} \sum_{\{S\}} \prod_{<i j>}\left(1+t S_{i} S_{j}\right)\right]^{p},
$$

where we have divided out various factors of two and $\cosh \beta$ in order to simplify the formulae later on. $N$ is the number of vertices in graph $G$. Note that there is no vertex and are no spins at the end of the root, so that the product over links does not include the root link. In fact we shall consider a generalization of this model that has $p$ coupling constants. Expanding out the above equation gives, with the extra coupling constants,

$$
\begin{gathered}
\mathcal{Z}^{I}(x,\{\lambda\})=\sum_{G \in \mathcal{G}_{r}^{I}} x^{N} \frac{1}{2^{N p}} \sum_{\{S\}} \prod_{<i j>}\left[1+\lambda_{1} \sum_{\alpha=1}^{p} S_{i}^{\alpha} S_{j}^{\alpha}+\lambda_{2} \sum_{\alpha=1}^{p-1} \sum_{\beta=\alpha+1}^{p} S_{i}^{\alpha} S_{j}^{\alpha} S_{i}^{\beta} S_{j}^{\beta}+\cdots\right. \\
\left.+\lambda_{p} \prod_{\alpha=1}^{p} S_{i}^{\alpha} S_{j}^{\alpha}\right]
\end{gathered}
$$


where $S_{i}^{\alpha}$ is the flavour $\alpha$ spin on vertex $i$, the second summation is over all spin configurations and $\{\lambda\}$ is the set of coupling constants $\left\{\lambda_{1}, \lambda_{2}, \cdots, \lambda_{p}\right\}$, with $0 \leq \lambda_{j} \leq 1$ for all $j$. Of course by setting $\lambda_{j}=t^{j}$ one recovers the ordinary model (87). Alternatively by setting $\lambda_{j}=0$ for $j>1$ we recover the $\mathrm{O}(\mathrm{n})$ models studied in 14, 15] with $n=p$. To save writing we define $T \equiv \mathcal{Z}^{I}(x,\{\lambda\})$. We will show that $T$ satisfies $T=f(T)$ where

$$
f(y)=\sqrt{1-2 x y} \mathcal{Z}^{I I}\left(x^{\prime},\left\{\lambda^{\prime}\right\}\right)+x y^{2},
$$

with

$$
x^{\prime}=x(1-2 x y)^{-\frac{3}{2}}, \quad \quad \lambda_{j}^{\prime}=\lambda_{j} \frac{(1-2 x y)}{\left(1-2 x y \lambda_{j}\right)} ;
$$

$\mathcal{Z}^{I I}\left(x^{\prime},\left\{\lambda^{\prime}\right\}\right)$ is defined as in (88), but using the set of rooted 1PI graphs, $\mathcal{G}_{r}^{I I}$. In a similar fashion to (2) the function $f(y)$ takes a 1PI graph and glues trees on to it, but this time as well as $x$ being changed to $x^{\prime}$, the coupling constants are also renormalized. Note that a similar renormalization is studied in [8].

To justify the above equations let us first consider the case $p=1$. For each link we have a factor such as $\left(1+\lambda_{1} S_{i} S_{j}\right)$ and when we multiply these factors together, the sum over spins will cause any terms containing odd powers of a given spin to vanish. The only non-vanishing terms correspond to sets of closed loops; we shall refer to such a nonvanishing term as a loop configuration. Thus for each graph $G$, we sum over all ways of drawing sets of closed non-intersecting, non-backtracking loops on the graph (call this set of loop configurations $\mathcal{L}_{G}$ ). So,

$$
\mathcal{Z}^{I}\left(x, \lambda_{1}\right)=\sum_{G \in \mathcal{G}_{r}^{I}} x^{N} \sum_{\mathcal{L} \in \mathcal{L}_{G}} \lambda_{1}^{l}
$$

where $N$ is the number of vertices in graph $G$, and $l$ is the number of links making up the loops in the loop configuration $\mathcal{L}$.

As before, we will make the graphs $G$ (where $G \in \mathcal{G}_{r}^{I}$ ) by gluing together 1PI blobs (fig. 1). Note that because of the tree-like structure, any given closed loop is wholly contained within a single blob. This means that the partition function for a given graph $G$, factorizes into a product of contributions from the individual blobs. To calculate the transfer function, $f(y)$, we take a rooted 1PI blob, summing over all graphs and loop configurations, as well as over all ways of attaching trees to the blob. If no trees were attached, one would just have $\mathcal{Z}^{I I}\left(x, \lambda_{1}\right)$, which is

$$
\mathcal{Z}^{I I}\left(x, \lambda_{1}\right)=\sum_{G \in \mathcal{G}_{r}^{I I}} x^{N} \frac{1}{2^{N}} \sum_{\{S\}} \prod_{<i j>}\left[1+\lambda_{1} S_{i} S_{j}\right]=\sum_{G \in \mathcal{G}_{r}^{I I}} x^{N} \sum_{\mathcal{L} \in \mathcal{L}_{G}} \lambda_{1}^{l} 1^{L-l},
$$

where $L$ is the number of internal links in graph $G$ (i.e. excluding the root link); that is, $L=\frac{1}{2}(3 N-1)$. Attaching a tree weighted with $y$, gives a factor of $2 x y$ (there is an extra vertex and there are two ways of hanging the tree off the link). For a given $G$ and $\mathcal{L}$, 
each link either contributes $\lambda_{1}$ if it is part of a closed loop or 1 if it is not. Attaching an arbitrary number of trees to an internal link that contributed 1 changes this contribution to $(1-2 x y)^{-1}$; however for those that contributed $\lambda_{1}$ we get $\lambda_{1}\left(1-2 x y \lambda_{1}\right)^{-1}$, since adding a vertex on a link which was part of a closed loop, increases the length of that loop by one and hence gives an extra factor of $\lambda_{1}$. Thus summing over all ways of attaching trees causes the change:

$$
\left(1+\lambda_{1} S_{i} S_{j}\right) \longrightarrow \frac{1}{1-2 x y}\left(1+S_{i} S_{j} \frac{\lambda_{1}(1-2 x y)}{\left(1-2 x y \lambda_{1}\right)}\right)
$$

and hence

$$
\mathcal{Z}^{I I}\left(x, \lambda_{1}\right) \longrightarrow \sqrt{1-2 x y} \mathcal{Z}^{I I}\left(x^{\prime}, \lambda_{1}^{\prime}\right)
$$

The extra term in (89) comes from the case in which the blob is just a single vertex.

The general case for $p>1$ follows with only minor modifications to the argument. Each closed loop is labelled with a spin flavour (an integer $\alpha$, with $1 \leq \alpha \leq p$ ) and loops with different spin flavours can intersect. Thus for a given graph $G$ and loop configuration $\mathcal{L}$, a given link will have $j$ spin flavours running through it $(0 \leq j \leq p)$, giving a corresponding factor of $\lambda_{j}$. Adding an arbitrary number of trees to this link changes the contribution to $\lambda_{j}\left(1-2 x y \lambda_{j}\right)^{-1}$. Hence, we get (89) and (90) for the general case of a rooted 1PI blob with trees, each weighted by $y$, hanging off it. Setting $y=T$, we recover the partition function for graphs with $G \in \mathcal{G}_{r}^{I}$, that is, $T=f(T) ; f(y)$ is the transfer function for the full model.

\subsection{Exponential decay of blobs}

As before, we define distance to be the geodesic distance, but count only the links connecting 1PI blobs. Again one has $G_{r}=f^{(r)}(v T)$, giving the partition function for a model where all blobs at distance $r$ are weighted by $v$. The average number of 1PI blobs at distance $r$ is

$$
\langle n\rangle_{r}=\left[\frac{v}{G_{r}} \frac{\partial G_{r}}{\partial v}\right]_{v=1}=\left.\frac{1}{T} \frac{\partial G_{r}}{\partial v}\right|_{v=1}
$$

Since $G_{r}=f\left(G_{r-1}\right)$,

$$
\langle n\rangle_{r}=\left.\left.\frac{1}{T} \frac{\partial f}{\partial y}\right|_{y=T} \frac{\partial G_{r-1}}{\partial v}\right|_{v=1}=\left.\frac{\partial f}{\partial y}\right|_{T}\langle n\rangle_{r-1}
$$

so that $\langle n\rangle_{r}=B^{r}$ with $B=\left.\frac{\partial f}{\partial y}\right|_{T}$. Thus we still have an exponential decay of the average number of blobs with distance and

$$
B=\frac{x T}{1-2 x T}\left[1-3 x T+\frac{(1-x T)}{\mathcal{Z}^{I I}}\left(3 x^{\prime} \frac{\partial \mathcal{Z}^{I I}}{\partial x^{\prime}}+\sum_{j=1}^{p} 2 \lambda_{j}^{\prime}\left(\lambda_{j}^{\prime}-1\right) \frac{\partial \mathcal{Z}^{I I}}{\partial \lambda_{j}^{\prime}}\right)\right]_{y=T},
$$


where $\mathcal{Z}^{I I}=\mathcal{Z}^{I I}\left(x^{\prime},\left\{\lambda^{\prime}\right\}\right)$. One can also show that

$$
\begin{aligned}
\left(\frac{\partial T}{\partial x}\right)_{\{\lambda\}} & =\left.\frac{1}{(1-B)}\left(\frac{\partial f(y)}{\partial x}\right)_{\{\lambda\}}\right|_{y=T} \\
& =\frac{T}{x(1-B)}\left[B-x T+(1-x T) M^{I I}\left(x^{\prime},\left\{\lambda^{\prime}\right\}\right)\right] \\
& \sim\left(x_{c}-x\right)^{-\gamma_{s t r}}
\end{aligned}
$$

where

$$
M^{I I}\left(x^{\prime},\left\{\lambda^{\prime}\right\}\right) \equiv \frac{x^{\prime}}{\mathcal{Z}^{I I}}\left(\frac{\partial \mathcal{Z}^{I I}}{\partial x^{\prime}}\right)_{\left\{\lambda^{\prime}\right\}} .
$$

Thus as in the previous case, at $x_{c}, B_{c}=1$ in the tree phase and on its boundary, where $\gamma_{s t r} \geq 0$, and we have $0<B_{c}<1$ in the magnetized phase. Using equations (97) and (99) one can show, barring accidental cancellations, that $\gamma_{s t r}=\frac{1}{2}$ in the tree phase and that

on the boundary, $\gamma_{s t r}^{*}=\gamma^{I I} /\left(\gamma^{I I}-1\right)$, which is just Durhuus' formula 8$]$; where $\gamma^{I I}\left(\left\{\lambda^{\prime}\right\}\right)$, which is negative, is the value of the string susceptibility for $\mathcal{Z}^{I I}\left(x^{\prime},\left\{\lambda^{\prime}\right\}\right)$, that is,

$$
\left(\frac{\partial^{2} \mathcal{Z}^{I I}}{\partial x^{\prime 2}}\right)_{\left\{\lambda^{\prime}\right\}} \sim\left(x_{c}^{\prime}\left(\left\{\lambda^{\prime}\right\}\right)-x^{\prime}\right)^{-\left(1+\gamma^{I I}\left(\left\{\lambda^{\prime}\right\}\right)\right)} .
$$

\subsection{The spin-spin correlation function}

Next we consider the spin-spin correlation function. Rather than fixing all the spins in the root blob, as we did for the toy model, it is more convenient to add a set of $p$ external spins on the root, which are all fixed to be +1 . The partition function is as in (88), but with an extra factor for the link connecting the external spins to the root blob, which we shall denote $W_{\text {ext }}$ (we will not add an extra factor of $x$ ). Thus,

$$
\mathcal{Z}^{I}(x,\{\lambda\})=\sum_{G \in \mathcal{G}_{r}^{I}} x^{N} \frac{1}{2^{N p}} \sum_{\{S\}}\left[W_{e x t} \prod_{<i j>} W_{i j}\right],
$$

where the weight $W_{i j}$ for link $\langle i j>$ is given by the square bracket in equation (88). Note that when the sum over spins is performed, the factor $W_{\text {ext }}$ just gives a contribution of one, so that the function $\mathcal{Z}^{I}$ is unchanged by adding the external spins. The average total spin at distance $r$ is

$$
\langle\Sigma S\rangle_{r}^{+}=\frac{1}{\mathcal{Z}^{I}} \sum_{G \in \mathcal{G}_{r}^{I}} x^{N} \frac{1}{2^{N p}} \sum_{\{S\}}\left[W_{e x t}\left(\prod_{<i j>} W_{i j}\right) \sum_{k, \alpha} S_{k}^{\alpha}\right],
$$

where the last summation is over all spins, $k$, at distance $r$ and all spin flavours, $\alpha$.

Consider $\mathcal{Z}^{I}\langle\Sigma S\rangle_{r}^{+}$, the only non-zero contributions come from the configurations in which there is a flavour $\alpha$ line from the external spins to vertex $k$, in addition to the usual 
sets of closed loops. Define $\bar{G}_{r}$ by

$$
\mathcal{Z}^{I}\langle\Sigma S\rangle_{r}^{+}=p \sum_{G \in \mathcal{G}_{r}^{I}} x^{N} \frac{1}{2^{N p}} \sum_{\{S\}}\left[W_{e x t}\left(\prod_{<i j>} W_{i j}\right) \sum_{k} S_{k}^{1}\right]=p \bar{G}_{r}
$$

Then $\bar{G}_{r}$ is a sum over all rooted graphs and all loop configurations that have a flavour 1 line from the external spins to a vertex $k$ at distance $r$; the location of the vertex $k$ is also summed over. However, we can write $\bar{G}_{r}$ in terms of $\bar{G}_{r-1}$. This is shown diagrammatically in fig. 4, where links included in the flavour 1 line are drawn thicker than those which are not included. The symbol on the left-hand side is used to represent $\bar{G}_{r}$, it has a flavour 1 line of length $r$, note that we are still measuring distances from the root blob (not from the external spins).

Figure 4: Formula for $\bar{G}_{r}$

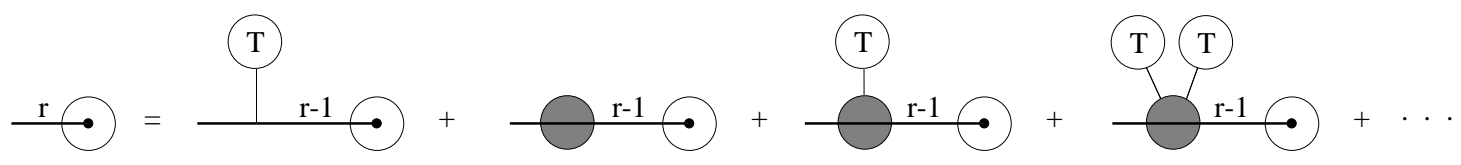

That is,

$$
\bar{G}_{r}=\left[2 \lambda_{1} x T+\mathcal{Z}_{2}^{I I}\left(x^{\prime},\left\{\lambda^{\prime}\right\}\right) \lambda_{1}(1-2 x T)\right] \bar{G}_{r-1},
$$

where $\mathcal{Z}_{2}^{I I}(x,\{\lambda\})$, which is drawn as a shaded circle with a thick line passing through it, is the partition function for 1PI graphs with two distinct legs and a flavour 1 line running through (it does not include factors of $\lambda_{1}$ on the two legs); $x^{\prime}$ and $\left\{\lambda^{\prime}\right\}$ are given by (90) evaluated at $y=T$. Define $D$ to be equal to the square bracket above, then $\bar{G}_{r}=D^{r} \bar{G}_{0}$. So that we have an exponential decay with distance,

$$
\langle\Sigma S\rangle_{r}^{+}=\frac{p}{\mathcal{Z}^{I}} D^{r} \bar{G}_{0}=D^{r}\langle\Sigma S\rangle_{0}^{+}
$$

The total average spin is

$$
\langle\Sigma S\rangle^{+}=\langle\Sigma S\rangle_{0}^{+} \frac{1}{1-D} .
$$

The magnetization in the grand canonical ensemble is given by

$$
\mathcal{M}=\frac{\langle\Sigma S\rangle^{+}}{p\langle N\rangle}=\frac{\langle\Sigma S\rangle_{0}^{+}}{p\langle N\rangle_{0}}\left(\frac{1-B}{1-D}\right)=\mathcal{M}_{0}\left(\frac{1-B}{1-D}\right),
$$

where $\mathcal{M}_{0}$ is the magnetization of the root blob $\left(0 \leq \mathcal{M}_{0} \leq 1\right)$. We have used the fact that the average number of vertices at distance $r$ is $\langle N\rangle_{r}=B^{r}\langle N\rangle_{0}$, so that $\langle N\rangle=$ $\langle N\rangle_{0} /(1-B)$. This is easily shown by defining $G_{r}^{\prime}=f^{(r)}\left(\left.f(T)\right|_{x z}\right)$ and following the derivation of (33). Note that, at $x_{c}$, in the tree phase $B_{c}=1$, so that $\mathcal{M}=0$ throughout this phase as one might expect. 
In the next section it is assumed that $D<1$, that is, that the spin-spin correlation length does not diverge; the easiest way to justify this would be to show that $D<B$, as we already know that $B \leq 1$. The following calculation will make this assumption plausible. From (107) one clearly has $D \leq B$, (at least provided that $\langle\Sigma S\rangle_{0}^{+} \neq 0$ ), since $\langle\Sigma S\rangle_{r}^{+} \leq p\langle N\rangle_{r}$. To improve this inequality we need an equation for $B$ which is simpler than (97). Define $G_{r}^{\prime \prime}$ to be the partition function for rooted graphs, with a marked vertex at distance $r$ - it is a sum over all graphs, $G \in \mathcal{G}_{r}^{I}$, all loop configurations and all ways of marking a vertex at distance $r$; that is, $G_{r}^{\prime \prime}=T\langle N\rangle_{r}$. Then following the derivation of (106) we have

$$
G_{r}^{\prime \prime}=\left[2 x T+\mathcal{Z}_{t}^{I I}\left(x^{\prime},\left\{\lambda^{\prime}\right\}\right)(1-2 x T)\right] G_{r-1}^{\prime \prime}=B G_{r-1}^{\prime \prime},
$$

where $\mathcal{Z}_{t}^{I I}$ is the partition function for 1PI graphs, with two distinct legs — it is a sum over all such graphs and all loop configurations. Note, $\mathcal{Z}_{t}^{I I}$ differs from $\mathcal{Z}_{2}^{I I}$ in that there is no flavour 1 line running through it. Thus,

$$
\begin{aligned}
& B=2 x T+\mathcal{Z}_{t}^{I I}\left(x^{\prime},\left\{\lambda^{\prime}\right\}\right)(1-2 x T) \\
& D=\lambda_{1}\left[2 x T+\mathcal{Z}_{2}^{I I}\left(x^{\prime},\left\{\lambda^{\prime}\right\}\right)(1-2 x T)\right] \text {. }
\end{aligned}
$$

Now, if one could show that $\mathcal{Z}_{t}^{I I}\left(x^{\prime},\left\{\lambda^{\prime}\right\}\right) \geq \mathcal{Z}_{2}^{I I}\left(x^{\prime},\left\{\lambda^{\prime}\right\}\right)$, then this would imply that $D \leq \lambda_{1} B$.

Consider $\mathcal{Z}_{+-}^{I I}\left(x^{\prime},\left\{\lambda^{\prime}\right\}\right)$, the partition function for 1PI graphs with two distinct legs, in which the flavour 1 spins on the two vertices connected to the legs are held fixed at +1 and -1 respectively; all other spins are summed over. Then one can easily see that $\mathcal{Z}_{+-}^{I I}\left(x^{\prime},\left\{\lambda^{\prime}\right\}\right)=\mathcal{Z}_{t}^{I I}\left(x^{\prime},\left\{\lambda^{\prime}\right\}\right)-\mathcal{Z}_{2}^{I I}\left(x^{\prime},\left\{\lambda^{\prime}\right\}\right)$, since the only non-vanishing terms correspond either to sets of closed loops, or configurations in which there is also a flavour 1 line running between the two fixed spins; this last set of terms is multiplied by the product of the fixed spins, namely -1 . For small enough values of $\left\{\lambda^{\prime}\right\}, \mathcal{Z}_{+-}^{I I}\left(x^{\prime},\left\{\lambda^{\prime}\right\}\right)$ is manifestly positive; the weights $W_{i j}$ for each link will be positive for any spin configuration (see equation (88)), and hence $\mathcal{Z}_{+-}^{I I}$ is just the sum of positive terms. At $\lambda_{j}^{\prime}=1$ for all $j$, the system is completely magnetized and hence $\mathcal{Z}_{+-}^{I I}=0$. One would expect that $\mathcal{Z}_{+-}^{I I}\left(x^{\prime},\left\{\lambda^{\prime}\right\}\right)>0$ for other values of $\left\{\lambda^{\prime}\right\}$. In which case $D \leq \lambda_{1} B$, with $D<B$ in general and $D=B$ only when $\lambda_{j}=\lambda_{j}^{\prime}=1$ for all $j$. Thus in general $D<1$ and the spinspin correlation length does not diverge. Note that our definition of distance is only good in the tree-like phase and on its boundary, and hence this result does not say anything about the possible divergence of the proper spin-spin correlation length (defined using the geodesic distance) at the magnetization transition, between the $\mathrm{U}$ and $\mathrm{M}$ phases (see figure 5).

Figure 5 shows a tentative phase diagram for the standard model defined by (87); $\mathrm{T}$ is the (unmagnetized) tree-like or branched polymer phase, $\mathrm{U}$ the unmagnetized phase 
and $M$ the magnetized phase. See [12, 13] for a discussion of various possible alternative phase diagrams. Each line of this phase diagram for constant $p$ (where $p$ is a non-negative integer) corresponds to a line through the $p$-dimensional phase space of the generalized model. Within the $\mathrm{U}$ and $\mathrm{M}$ phases the model behaves in a similar fashion to the pure gravity case and it seems probable that $\gamma_{s t r}=-\frac{1}{2}$ throughout both these phases. The transition between the magnetized (M) and unmagnetized (U) phases is caused by $\mathcal{M}_{0}$ vanishing as the coupling constants $\{\lambda\}$ are varied. From the KPZ results, we expect to have $-\frac{1}{2} \leq \gamma_{s t r}^{*} \leq 0$ along the corresponding critical line, at least for $p \leq 2$. The tree-like phase has $\gamma_{s t r}=\frac{1}{2}$ throughout, the generic value for branched polymers.

Figure 5: Tentative phase diagram

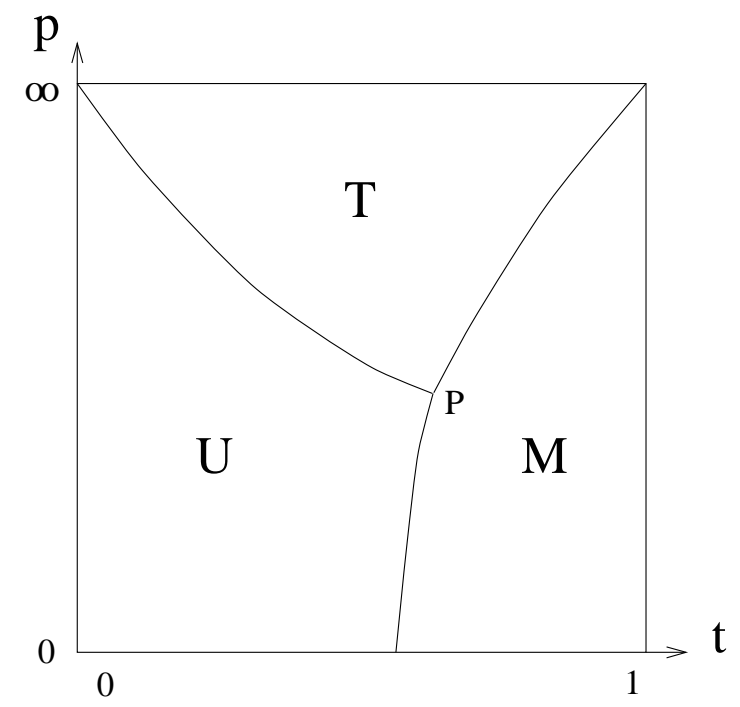

\subsection{Critical exponents}

Now consider the transition from the magnetized to the tree phase, which is caused by $B_{c} \rightarrow 1$. The behaviour of the full model at this transition is very similar to that of the toy model. Following the analysis in section [3, we define a mass by $m=-\ln B$. As before there is an exponential decay, so we will take $\eta=1$, which gives $\gamma_{s t r}=\nu$ provided that the scaling relation $\gamma_{s t r}=\nu(2-\eta)$ holds. Consider the magnetic exponents, at $x_{c}$ and fixed integer $p$ we vary the $\{\lambda\}$ in order to approach the phase transition; the variable $\Delta \lambda$ will be used to parameterize this, with $\Delta \lambda=0$ at the transition. Then by definition $m \sim(\Delta \lambda)^{\nu_{m}}$, but $m=-\ln B_{c}$ so that $B_{c} \sim 1-(\Delta \lambda)^{\nu_{m}}$. Now $\beta_{m}$ is defined by $\mathcal{M} \sim(\Delta \lambda)^{\beta_{m}}$, but $\mathcal{M} \sim 1-B_{c}$ from (109), thus $\beta_{m}=\nu_{m}$; note, we have assumed that we are not close to any point at which $\mathcal{M}_{0}$ vanishes ( $P$ in fig. 5 is such a point). It has also been assumed that $D_{c} \neq 1$ at the transition, i.e. that the spin-spin correlation length does 
not diverge; this was justified in the previous section. If we assume that $\gamma_{m}=\nu_{m}\left(2-\eta_{m}\right)$ with $\eta_{m}=1$, then we have $\gamma_{m}=\nu_{m}=\beta_{m}$. Assuming the scaling relations $2-\alpha=\nu_{m} d_{H}^{*}$ and $\alpha+2 \beta_{m}+\gamma_{m}=2$, gives $d_{H}^{*}=3$. The calculation in [11], which gave that $\nu d_{H}=1$, still applies, so that $\nu^{*}=\frac{1}{3}$ and hence $\gamma_{s t r}^{*}=\frac{1}{3}$. This is encouraging as the analysis in [8] leads one to suppose that $\gamma_{s t r}^{*}=\frac{1}{3}$ provided $\gamma_{s t r}=-\frac{1}{2}$ in the magnetized phase. Also, applying the relation $\beta_{m} \delta_{m}=\beta_{m}+\gamma_{m}$ gives $\delta_{m}=2$.

Now $B$ is given by (97) and in the magnetized phase at $x_{c}, x^{\prime}=x_{c}^{I I}\left(\left\{\lambda^{\prime}\right\}\right)$. Provided that we are away from points at which $\mathcal{M}_{0}$ vanishes, $\mathcal{Z}^{I I}\left(x^{\prime},\left\{\lambda^{\prime}\right\}\right)$ should be analytic and Taylor expandable about $\left\{\lambda^{\prime *}\right\}$ (the value of $\left\{\lambda^{\prime}\right\}$ at the $\mathrm{M}$ to $\mathrm{T}$ phase transition). Assuming that $x_{c}(\{\lambda\}) T_{c}(\{\lambda\})$ is expandable in powers of $\Delta \lambda$ as far as $\Delta \lambda$, then barring accidental cancellations $B_{c} \sim 1-\Delta \lambda$ and hence $\nu_{m}=\beta_{m}=\gamma_{m}=1$ and $\alpha=-1$.

Thus on the assumption of various scaling relations and that our definitions of $m$ and $\mathcal{M}$ are adequate, and barring problems such as accidental cancellations, the set of exponents given in table 1 should also apply for the full model for the $\mathrm{M}$ to $\mathrm{T}$ transition, away from point $P$. Again it is not clear what the value of $d_{H}$ is, within the $\mathrm{M}$ phase; however one might guess that $d_{H}=4$, the pure gravity value [11, 16, 17], throughout the $\mathrm{U}$ and $\mathrm{M}$ phases (in which case it is probable that $\nu=1 / 4$ and $\eta=4$ here). Note that the mechanism by which the $U$ to $T$ transition occurs is essentially the same and so we would expect to have the same set of exponents, although the magnetic exponents are not relevant since $\mathcal{M}=0$ in both the $\mathrm{U}$ and $\mathrm{T}$ phases. Perhaps one should note that a lowtemperature expansion matrix model calculation in the limit $c \rightarrow \infty$ 四 gives that $\gamma_{s t r}^{*}=\frac{1}{3}$ at the transition between the tree and magnetized phases, and that the truncated model studied in [12], which is supposed to approximate the $\mathrm{U}$ to $\mathrm{T}$ transition, has $\gamma_{s t r}^{*}=\frac{1}{3}$ and $\alpha=-1$, agreeing with our predictions. In addition the q-state Potts model at large $q$ [5], which is equivalent to the multiple Ising model in the limit $q=p=\infty$ and may be related to it for finite $q$, has a branched polymer region (with $\gamma_{s t r}=\frac{1}{2}$ ) separating two pure gravity regions (with $\gamma_{s t r}=-\frac{1}{2}$ ) and has $\gamma_{s t r}^{*}=\frac{1}{3}$ and $\alpha=-1$ at both transitions.

At point $P$, the above argument fails since $\mathcal{M}_{0} \rightarrow 0$ and hence it is possible to have $\beta_{m} \neq \nu_{m}$; some of the other assumptions may break down here too. Thus at this point one might expect to get a different set of exponents. In any case the calculations in this section only apply for non-negative integer $p$ and it is not entirely clear whether $p^{*}$, the value of $p$ at point $P$, is an integer. It is tempting to suppose that $p^{*}=2$, in which case one could understand the breakdown of the KPZ formula in terms of the appearance of the branched polymer phase, however Monte Carlo simulations do not seem to support this hypothesis [3]. 


\section{Conclusion}

In this article we have studied correlation functions in two-dimensional quantum gravity coupled to Ising spins. For the toy model two approximations are used: (i) the spin configurations allowed are the ones dominant in the low temperature expansion of multiple Ising spins on dynamically triangulated surfaces and (ii) the distance between two spins is identified with the number of links separating 1PI subgraphs along a path connecting the spins. In the full model only the second approximation is made. We do not expect this last approximation to be important for values of $\beta$ where $\gamma_{s t r}(\beta)>0$, i.e. for values of $\beta$ where the average number of vertices of a generic $\phi^{3}$ graph diverges for $x \nearrow x_{c}(\beta)$, since the average number of vertices in an 1PI part of a generic $\phi^{3}$ graph in our ensemble is very small. However, if $\gamma_{s t r}(\beta)<0$ the average number of vertices in a generic $\phi^{3}$ graph is itself small, even at the critical point $x_{c}(\beta)$ and our definition of distance can not be used to extract in a reliable way the fractal properties of the ensemble of $\phi^{3}$ graphs. Approximation (i) has been shown to be exact in the $c \rightarrow \infty$ limit [4] and numerical simulations [3] suggest that it is an excellent approximation except for the smallest values of $\beta$ if many Ising spins are coupled to two-dimensional gravity. For moderate values of $c$ it is difficult to judge if approximation (i) is reliable all the way down to $\beta^{*}$. For $c=1 / 2$ and $c=1$ (i) not a good approximation for $\beta \in\left[0, \beta^{*}\right]$.

The toy model allows us to analyze the two-point function (the puncture-puncture correlator) and the spin-spin correlator as a function of the distance $r$. For $\beta \leq \beta^{*}$ we have $\gamma_{s t r}>0$ and the fractal structure is reliably extracted from the two-point function. We find that $d_{H}=2$ for $\beta<\beta^{*}$, while it jumps to $d_{H}^{*}=3$ at $\beta^{*}$. Whilst we can not calculate $d_{H}$ for $\beta>\beta^{*}$, one might expect it to equal four, the pure gravity value [11]. The fact that $d_{H}$ is different from 4 for $\beta \leq \beta^{*}$ is an indication of the very strong interaction between gravity and matter for $c>1$.

A further indication of the strong link between geometry and matter configurations present in the model is found in the scaling relations for the magnetic exponents. They are found from the behaviour of the two-point function $\langle n\rangle_{r}$ in the region $\beta \geq \beta^{*}$. The exponential decay of $\langle n\rangle_{r}$ at $x_{c}(\beta)$ as a function of the distance $r$ defines a length scale

$$
\xi(\beta) \sim \frac{1}{\beta-\beta^{*}},
$$

which diverges for $\beta \rightarrow \beta^{*}$ and allows us to define the magnetic exponents using the standard hyper-scaling relations (note that $\xi(\beta)=\infty$ for $\beta \leq \beta^{*}$ ). The same exponents are found, for the toy model, by a direct calculation in the canonical and grand canonical ensembles. Strictly speaking, it seems more natural to define magnetic scaling properties from one of the spin-spin correlators, i.e. either $\langle\Sigma S\rangle_{r}^{++\cdots+}$ or $\mathcal{M}_{r}$, however the corresponding correlation length does not diverge at $\beta^{*}$. This is an indication of the geometric 
nature of the transition.

For the full model, a summation over all spin configurations is performed and the only approximation made is in the measure of distance that is used. As before there is an exponential decay of both the two-point function and the spin-spin correlation function, and one can define, in the magnetized phase, a geometric correlation length, which diverges as the tree phase is approached. Based on the analysis in section 7.3 we do not expect the spin-spin correlation length, at least as we have defined it, to diverge at the magnetized to tree-like transition. Using the geometric length scale and the scaling relations, which have been shown to hold for the toy model, all the critical exponents are calculated for the branched polymer phase and its boundary, on the basis of a small number of assumptions. The exponents are the same as those for the toy model (see table 1) and again $d_{H}=2$ within the tree phase and $d_{H}$ is equal to three on the critical line, showing the strong interaction between the matter fields and the geometry.

Some interesting questions remain to be answered, such as what happens outside the branched polymer phase, in particular what the values of the geometric exponents and $d_{H}$ are, and whether there is a diverging length associated with the spin-spin correlation function at the magnetization transition (between the $\mathrm{U}$ and $\mathrm{M}$ phases). Unfortunately our measure of distance is inadequate in this region and it will require further work using probably the full geodesic distance in order to determine this. It would also be useful to locate point $P$ on the phase diagram, as it has been suggested [17, 3] that $p^{*}>2$, in which case there is an intermediate region, for $2<p<p^{*}$, between the KPZ regime and the branched polymer phase.

Finally, it should perhaps be noted that most of the considerations in this paper also apply to the $\mathrm{O}(\mathrm{n})$ models [14, 15], as these are just a special case of our generalized multiple Ising model.

\section{Acknowledgements}

MGH would like to acknowledge the support of the Royal Society through their European Science Exchange Programme.

\section{References}

[1] V.G.Knizhnik, A.M.Polyakov and A.B. Zamolodchikov, Mod. Phys. Lett. A3 (1988) 819

[2] J.Distler and H.Kawai, Nucl. Phys. B321 (1989) 509;

F.David, Mod. Phys. Lett. A3 (1988) 1651 
[3] C.F.Baillie and D.A.Johnston, Phys. Lett. B286 (1992) 44, hep-lat/9204003;

S.M.Catterall, J.B.Kogut and R.L.Renken, Phys. Lett. B292 (1992) 277, heplat/9206021;

J.Ambjørn, B.Durhuus, T.Jónsson and G. Thorleifsson, Nucl. Phys. B398 (1993) 568, hep-th/9208030;

M.Bowick, M.Falcioni, G.Harris and E.Marinari, Nucl. Phys. B419 [FS] (1994) 665, hep-th/9310136;

J.Ambjørn and G.Thorleifsson, Phys. Lett. B323 (1994) 7, hep-th/9312157;

J.-P.Kownacki and A.Krzywicki, Phys. Rev. D50 (1994) 5329, hep-th/9401143

[4] M.Wexler, Nucl. Phys. B410 (1993) 377, hep-th/9305041;

M.Wexler, Mod. Phys. Lett. A8 (1993) 2703, hep-th/9307120

[5] M.Wexler, Nucl. Phys. B438 (1995) 629, hep-th/9405134;

J.Ambjørn, G.Thorleifsson, M.Wexler, Nucl. Phys. B439 (1995) 187, hep-lat/9411034

[6] J.Ambjørn, B.Durhuus and T.Jonsson, Mod. Phys. Lett. A9 (1994) 1221, hepth/9401137

[7] T.Jónsson and J.F.Wheater, Phys. Lett. B345 (1995) 227, hep-th/9411013

[8] B.Durhuus, Nucl. Phys. B426 (1994) 203, hep-th/9402052

[9] H.Kawai, N.Kawamoto, T.Mogami and Y.Watabiki, Phys. Lett. B306 (1993) 19, hep-th/9302133

[10] E.Brézin, C.Itzykson, G.Parisi and J.B.Zuber, Commun. Math. Phys. 59 (1978) 35

[11] J.Ambjørn and Y.Watabiki, Nucl. Phys. B445 (1995) 129, hep-th/9501049

[12] M.G.Harris, "A Two Term Truncation of the Multiple Ising Model Coupled to 2d Gravity", hep-th/9502036

[13] M.G.Harris and J.F.Wheater, Nucl. Phys. B427 (1994) 111, hep-th/9404174

[14] I.K.Kostov, Mod. Phys. Lett. A4 (1989) 217;

I.K.Kostov and M.Staudacher, Nucl. Phys. B384 (1992) 459, hep-th/9203030

[15] B.Eynard and C.Kristjansen, Nucl. Phys. B455 (1995) 577, hep-th/9506193;

B.Eynard and C.Kristjansen, "More on the exact solution of the $\mathrm{O}(\mathrm{n})$ model on a random lattice ...", hep-th/9512052

[16] S.Catterall, G.Thorleifsson, M.Bowick and V.John, Phys. Lett. B354 (1995) 58, heplat/9504009 
[17] J.Ambjørn, J.Jurkiewicz and Y.Watabiki, Nucl. Phys. B454 (1995) 313, heplat/9507014 